\title{
Study of ZZ and Neutral Current 4-Fermion Production at LEP2
}

\author{
Hartmut Rick* for the LEP collaborations Aleph, Delphi, L3 and Opal \\ University of California in Riverside \\ E-mail: 'Hartmut.Rick@cern.ch'
}

ABstract: Recent results on neutral current 4-fermion processes from the four LEP experiments are reviewed, including ZZ production, single Z production as well as general neutral current 4-fermion processes. After the end of LEP running, preliminary results from the full LEP2 data set are available for most channels.

\section{1. $\mathrm{Z}$ boson pair production}

Data at center-of-mass energies above the threshold for $\mathrm{Z}$ boson pair production has been recorded by the LEP experiments during the years 1997-2000.

For purposes of combining their results, the four LEP experiments have agreed on a common definition of the $\mathrm{ZZ}$ cross section, which corresponds to the two $\mathrm{NC02}$ diagrams that are shown in Figure tributions from other diagrams and their interference with the $\mathrm{NC} 02$ diagrams are excluded. This definition coincides to a
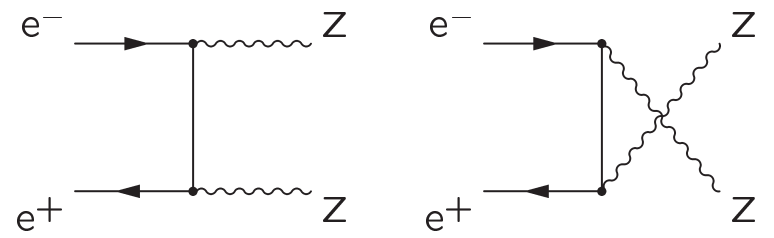

Figure 1: NC02 diagrams of Z boson pair production. large extent with typical experimental signal definitions that are based on the invariant masses of the fermion pairs. Inside the acceptance, contributions from other diagrams to the same final state and their interference with the NC02 diagrams are small, typically less than $10 \%$ of the ZZ rate. In order to produce a measured "NC02" cross section, the difference between the full contribution of all known processes and that of the NC02 diagrams alone is obtained from a Monte Carlo model and subtracted from the experimentally measured cross section.

The NC02 cross section can be precisely calculated in the framework of the Standard Model, it depends only on electroweak parameters like the mass and width of the $\mathrm{Z}$ boson, which are known with great precision. No free parameters need to be determined. The

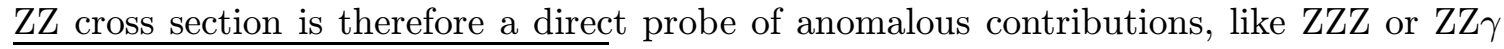

${ }^{*}$ Speaker. 
couplings or $\mathrm{TeV}$ scale quantum gravity. Limits on anomalous neutral gauge couplings are presented in another contribution to this conference [i]1]. Possible Higgs production would also contribute to the same final states.

Each of the four LEP experiments recorded around $680 \mathrm{pb}^{-1}$ of integrated luminosity above the threshold for ZZ production. With the ZZ cross section as predicted by the Standard Model, and taking into account the distribution of center-of-mass energies during LEP2 running, this corresponds to 560 pairs of $\mathrm{Z}$ bosons that should have been produced at each of the four experiments.

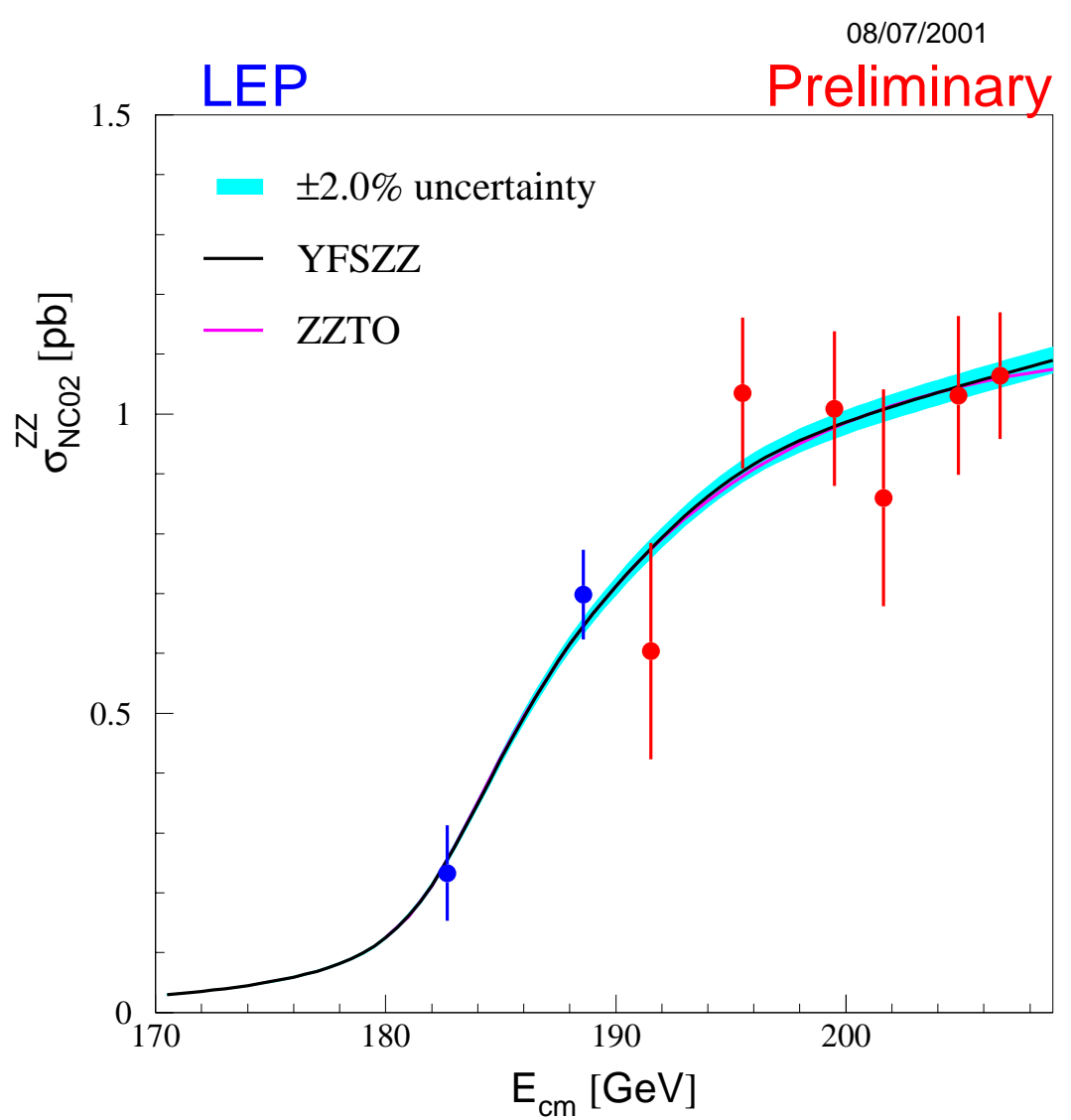

Figure 2: NC02 ZZ cross section, combined from the measurements of the 4 LEP experiments, as a function of center-of-mass energy of the accelerator. The final measurements at center-of-mass energies up to $189 \mathrm{GeV}$ have been published by all 4 collaborations, the results at higher energies are based on preliminary data. The measurement is compared to calculations of the two models

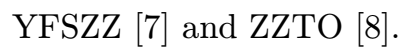

Not all of these events can be selected in a detector, however. From all ZZ decay channels, the final state consisting of two jets and two charged leptons, corresponding to $14 \%$ of the full cross section, can be selected with good efficiency and highest purity of up to $90 \%$. The missing energy channel, a pair of jets and an invisible decay of one of the $\mathrm{Z}$ bosons, has somewhat higher background contamination, but also a higher branching ratio of about $28 \%$. The channel with the highest branching fraction is the fully hadronic final state. It has about $49 \%$ of all $\mathrm{ZZ}$ decays, but suffers a large background from hadronic 
decays of $\mathrm{W}$ pairs. The purity in this channel can be improved by identifying jets originating from b quark decays, at the expense of losing those $60 \%$ of events without b quarks.

Each of these three classes of decay channels contributes with similar significance to a $\mathrm{ZZ}$ cross section measurement. The fully leptonic channels contribute only little, despite their clear signature, because of the small branching ratio.

The combined ZZ cross section measurement of the LEP experiments is shown in Figure $\overline{2}$. It agrees well with the Standard Model expectation, represented by calculations of the YFSZZ [i] models.

The results of the individual measurements and more details of the analyses are

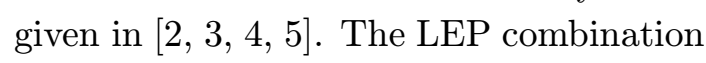
procedure is described in [i, $\overline{6}]$.

The L3 and Opal collaborations have also derived, from the ZZ data, the branching ratio of the $\mathrm{Z}$ boson into a pair of b quarks. Apparent deviations from the branching fraction which was previously measured at LEP1 energies would be ex-

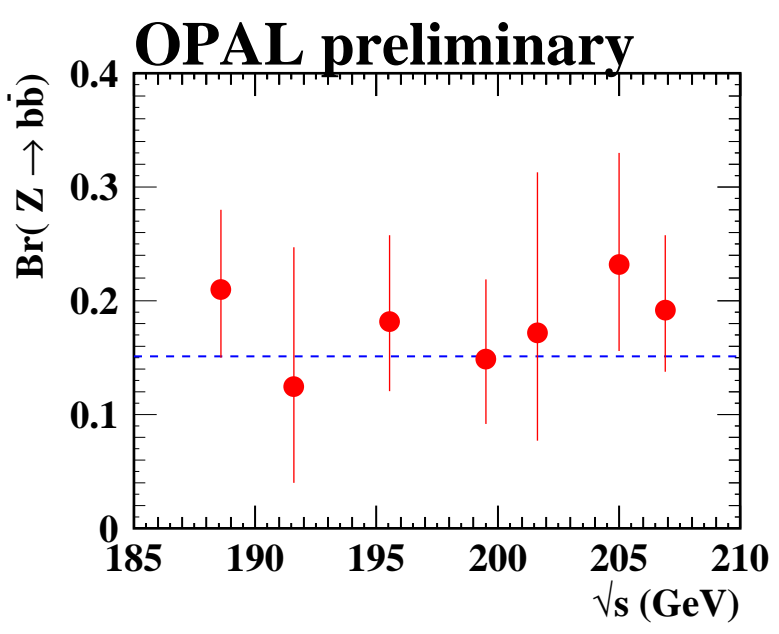

Figure 3: Branching fraction of $\mathrm{Z} \rightarrow \mathrm{b} \overline{\mathrm{b}}$ as obtained from $\mathrm{ZZ}$ data, as a function of center-ofmass energy. pected in the presence of contributions from Higgs boson production. As an example, the branching fraction measured by the Opal collaboration is shown in Figure 3 . The result is compatible with the expectation.

\section{General neutral current 4-fermion processes}

Measurements of neutral current 4-fermion processes can be extended outside the ZZ region. An analysis of a broad range of final states has been presented by the Delphi collaboration [9.9. With the cross section decreasing outside the $\mathrm{Z}$ resonance region, the purity of the event selection is generally worse than for ZZ production, but for certain final states or in special kinematic regions a meaningful analysis is still possible. As an example, a selection of $\mu^{+} \mu^{-} \mathrm{q} \overline{\mathrm{q}}$ events is shown in figure if $_{-}^{-}$Cross sections for this and other final states are given in $[\overline{\overline{9}}]$.

\section{Single-Z production}

So-called single-Z, or Zee production is a special case of neutral current 4-fermion processes, where an almost real photon is radiated off one of the beam electrons, and then scatters on the other electron, producing a $\mathrm{Z}$ boson in the final state, or a $\gamma^{\star}$, which decays into a pair of fermions. The first electron is scattered at very small angle and thus remains inside the beam hole and escapes detection. The second beam electron is scattered at a large angle, so that the visible final state consists of one electron and a pair of fermions in the detector. 

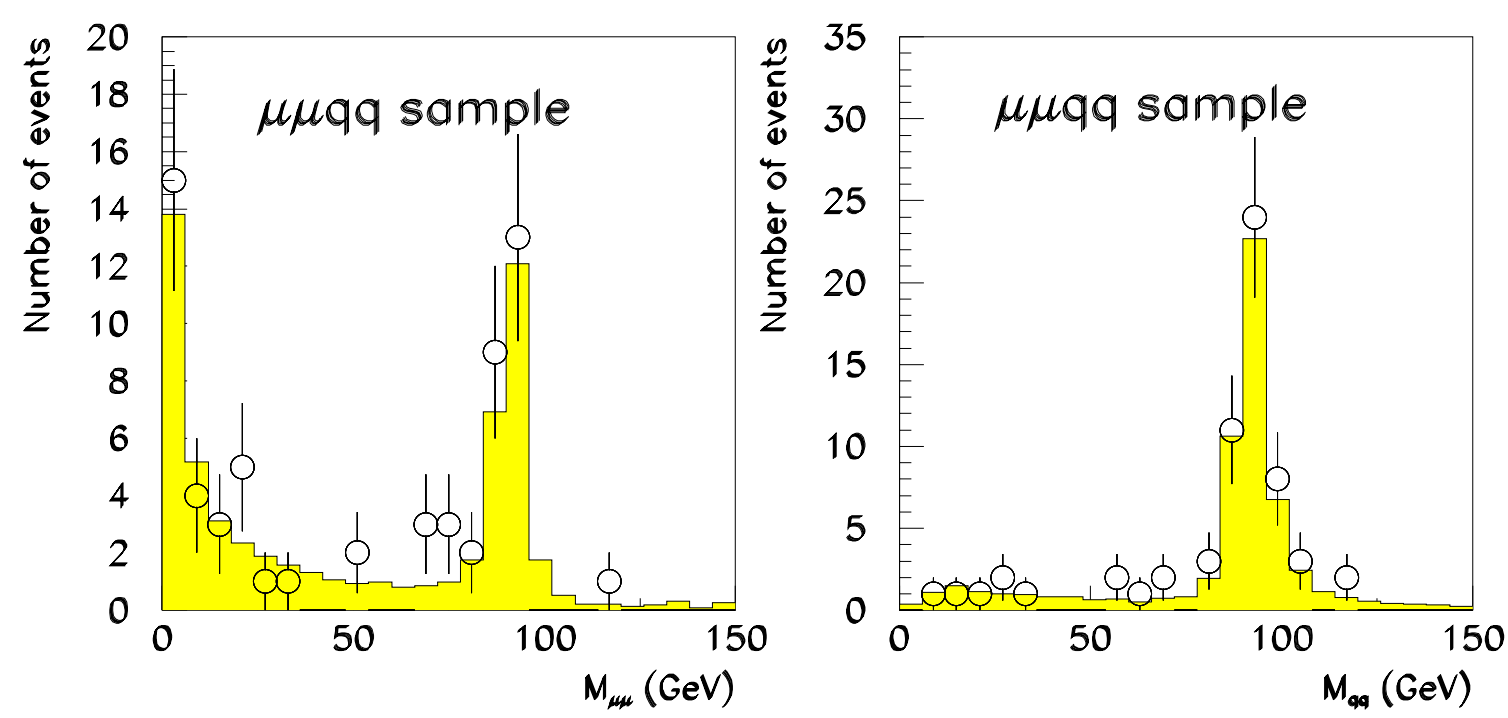

Figure 4: Invariant mass distribution of muon and jet pairs for 4 -fermion $\mu^{+} \mu^{-} \mathrm{q} \overline{\mathrm{q}}$ events. This preliminary result from the Delphi collaboration contains all available data recorded at center-ofmass energies between 183 and $208 \mathrm{GeV}$. The histogram is a fit to the data of separately normalised contributions from $\mathrm{ZZ}, \mathrm{Z} \gamma^{\star}$ and $\gamma^{\star} \gamma^{\star}$ processes, simulated with the Excalibur [i

Cross sections for jet and muon pairs have been measured by the Delphi collaboration [1] for all available high energy data.

Since the intermediate photon is almost real, the process can also be considered as a photon-electron collision. The Opal collaboration has measured the rate of jet pairs and

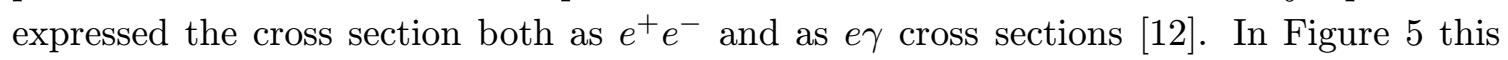
cross section is shown as a function of $\sqrt{\hat{s}}$, the center-of-mass energy of the er collision.

\section{Summary}

Neutral current 4-fermion processes have been studied at LEP2. Double resonant ZZ production has been examined by the LEP experiments as well as single resonant Zee production and general 4-fermion processes over wider fermion pair mass ranges. All observed rates are close to the respective Standard Model expectations.

\section{Acknowledgements}

The speaker would like to thank his colleagues from the LEP collaborations and in particular the members of the LEP-electroweak working group for providing their results and help for preparing this presentation.

\section{References}

[1] A. Oh, these proceedings.

[2] Aleph note 2001-006, March 8, 2001.

[3] Delphi note 2001-105 CONF 533, March 5, 2001. 


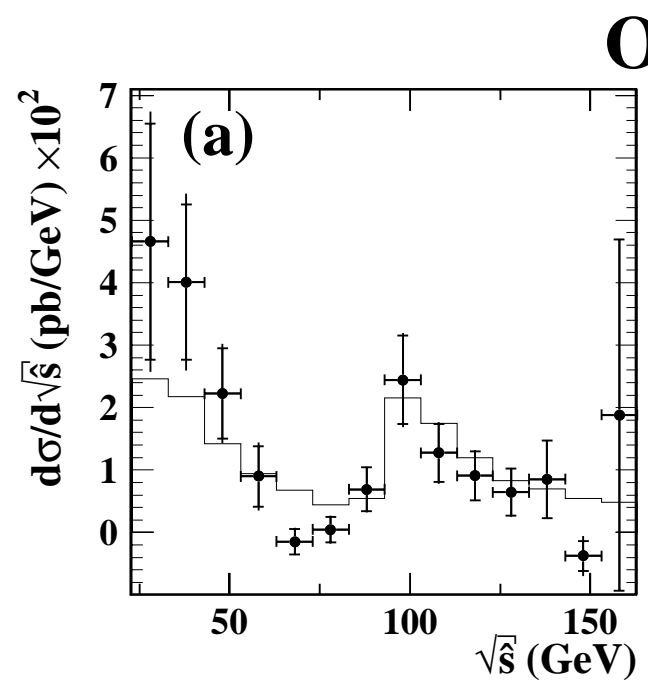

OPAL

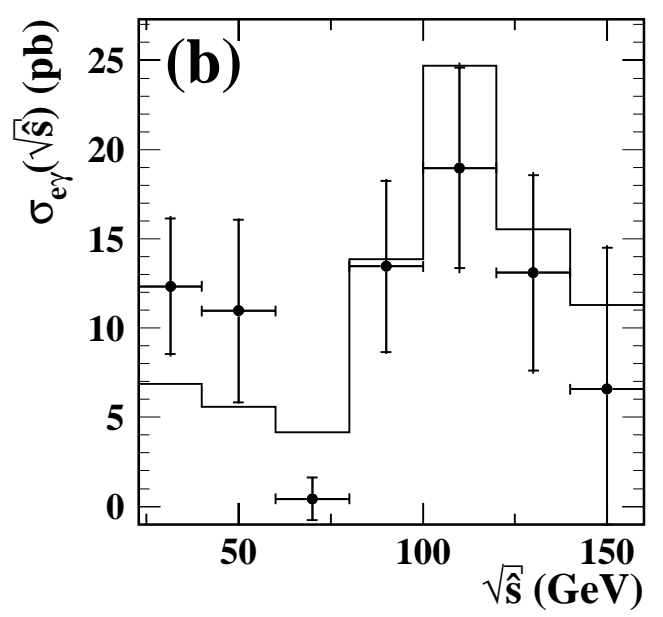

Figure 5: Zee cross section as a function of the $e \gamma$ center-of-mass energy, expressed as $e e$ cross section (a) and as er cross section (b). The data points are compared to the simulation of the grc4f [i] $\left[\overline{3}_{1}\right]$ Monte Carlo generator.

[4] L3 note 2696, July 4, 2001.

[5] Opal physics note PN482, July 7, 2001.

[6] LEP Electroweak Working Group note LEPEWWG/XSEC/2001-03, August 14, 2001.

[7] S. Jadach, W. Placzek, B.F.L. Ward, Phys. Rev. D56 (1997) 6939.

[8] G. Passarino, in M. Grünewald, G. Passarino, hep-ph/0005309.

[9] Delphi note 2001-096 CONF 524, June 25, 2001.

[10] F.A. Berends, R. Pittau, R. Kleiss, Comp. Phys. Commun. 85 (1995) 437.

[11] Delphi note 2001-100 CONF 528, June 10, 2001.

[12] Opal collaboration, G. Abbiendi et al., CERN-EP-2001-053, June 2001, hep-ex/0107047, submitted to Eur. Phys. J. C.

[13] J. Fujimoto et al., Comp. Phys. Commun. 100 (1997) 128. 